\title{
Spontaneous Unfolding and Refolding of Plantaricin $\alpha$-Helix in Molecular Dynamics Simulation
}

\author{
Shaomin Yan, Guang $\mathrm{Wu}^{*}$ \\ State Key Laboratory of Non-Food Biomass and Enzyme Technology, National Engineering Research Center for Non-Food \\ Biorefinery, Guangxi Key Laboratory of Bio-Refinery, Guangxi Academy of Sciences, Nanning, China \\ Email: ^hongguanglishibahao@gxas.cn
}

How to cite this paper: Yan, S.M. and Wu, G. (2019) Spontaneous Unfolding and Refolding of Plantaricin $\alpha$-Helix in Molecular Dynamics Simulation. Computational Molecular Bioscience, 9, 27-39. https://doi.org/10.4236/cmb.2019.91003

Received: January 29, 2019

Accepted: March 25, 2019

Published: March 28, 2019

Copyright () 2019 by author(s) and Scientific Research Publishing Inc. This work is licensed under the Creative Commons Attribution International License (CC BY 4.0).

http://creativecommons.org/licenses/by/4.0/

\section{c) (i) Open Access}

\begin{abstract}
Antimicrobial peptides are promising therapeutic agents in view of increasing resistance to conventional antibiotics. Antimicrobial peptides usually fold in $\alpha$-helical, $\beta$-sheet, and extended/random-coil structures. The $\alpha$-helical antimicrobial peptides are often unstructured in aqueous solution but become structured on bacterial membrane. The $\alpha$-helical structure allows the partitioning into bacterial membrane. Therefore it is important to understand the mechanism of unfolding and refolding of $\alpha$-helical structure in antimicrobial peptides. It is not very easy to obverse and study the process of unfolding and refolding of $\alpha$-helical antimicrobial peptides because of their rapidity. Therefore, molecular simulation provides a way to observe and explain this phenomenon. Plantaricin A is a 26 amino-acid antimicrobial pheromone peptide and can spontaneously unfold and refold under physiological condition. This study demonstrated the unfolding and refolding of plantaricin A by means of molecular simulation, and its mechanism was discussed with its implication to the Levinthal paradox.
\end{abstract}

\section{Keywords}

Alpha-Helix, Antimicrobial Peptides, Protein Folding, Plantaricin A

\section{Introduction}

Antimicrobial peptides are promising therapeutic agents, not only because they can act on Gram-positive/negative bacteria, protozoa, yeast, fungi, viruses, etc., but also because they come from almost all organisms [1] [2]. Besides having a great diversity in their function, antimicrobial peptides also have a great diversi- 
ty in their structures including $\alpha$-helical, $\beta$-sheet, extended/random-coil structure [3] [4] [5].

Of three thousands of antimicrobial peptides [6], $\alpha$-helical structure accounts $14.88 \%, \beta$-sheet structure accounts $2.69 \%$, whereas the majority $(58.85 \%)$ are unknown structures and the rest are mixed structures. Clearly, $\alpha$-helix is the most important structure in antimicrobial peptides. Although the net charge, amphipathicity and hydrophobicity, is the most important factor in antimicrobial peptides for their activity [3], the $\alpha$-helical structure produces distinct membrane-bound amphipathic conformation [7] and thus allows its partitioning into bacterial membrane.

Curiously, the $\alpha$-helical antimicrobial peptides are often unstructured in aqueous solution but become structured on bacterial membrane. Basically, the alternation between unfolding and refolding is not limited to $\alpha$-helical antimicrobial peptides because this feature is observed in diseases. The famous example is amyloid $\beta(\mathrm{A} \beta)$ peptide, whose $\alpha$-helix spontaneously unfolds under the physiological condition and the unfolding of amyloid $\beta$ is one of the causes for Alzheimer's disease [8]. Also, the unfolding and refolding are important in biotechnological settings, where microorganisms produce recombinant enzymes and biopharmaceutical proteins. For example, the refolding of secreted amylase in Bacillus subtilis is extremely important for a profitable production [9] [10].

Plantaricin A is an antimicrobial pheromone peptide produced by Lactobacillus plantarum and is composed of 26 amino acids. Plantaricin A has an $\alpha$-helix from position 11 to position 22, and this $\alpha$-helical structure can spontaneously unfold and refold under the physiological condition, because it is unstructured in water but becomes partly structured upon the exposure to micelles and fully structured under the physiological condition [11].

Because of importance of $\alpha$-helical structure in antimicrobial peptides and proteins, it is necessary to study the unfolding and refolding process in order to understand the underlying mechanism. Although it is not so easy to observe and study this process at small time scale, molecular dynamic simulation could provide a way to observe and explain this phenomenon. In this study, the unfolding and refolding of plantaricin A was found in molecular dynamic simulation and an attempt was made to discuss its mechanism.

\section{Materials and Methods}

\subsection{Data}

The 3D structure of plantaricin A is available at Protein Data Bank [12] identifier 1YTR, which contains 20 best structures from 100 calculated structures measured by $\mathrm{NMR}$ at $25^{\circ} \mathrm{C}, \mathrm{pH} 4$, zero ionic strength and ambient pressure. The selection of these 20 best structures was based on their lowest energy [13].

\subsection{Molecular Dynamics Simulation}

Of 20 best structures of 1YTR, the 20th structure was used for the molecular dy- 
namic simulation because it is also the one shown in various $3 \mathrm{D}$ visualization programs. Hydrogen atoms that did not appear in NMR measurement were added to plantaricin A using the Visual Molecular Dynamics Program [14].

The NAMD2 2.9 [15] was used to perform all the simulations with the all-atom force field CHARMM (v. 27) [16]. In simulations, the electrostatic interactions, the short-range non-bonded electrostatic and van der Waals interactions, and the long-range interactions were computed every $1 \mathrm{fs}, 2$ fs, and $4 \mathrm{fs}$; the particle-mesh Ewald was used with grid points no more than $\sim 1 \AA$ apart; the Langevin dynamics was coupled to all atoms except for the hydrogens with a 5 $\mathrm{ps}^{-1}$ damping coefficient; the Nose-Hoover Langevin piston was used with a decay period of $100 \mathrm{fs}$ and a damping time of $50 \mathrm{fs}$ under $1 \mathrm{~atm}$; a constant temperature was maintained; and $9 \AA$ was chosen for the distance between ions [17]. The final system size was 24,661 atoms. Each simulation was continued until the time that no change was observed in plantaricin A structures, usually $15 \mathrm{~ns}$.

Plantaricin A became unfolded in solution with ionic strengths of $150 \mathrm{mM}$ $\mathrm{NaCl}$ and $1.5 \mathrm{mM} \mathrm{CaCl}_{2}$ at $\mathrm{pH} 7.4$ and $37^{\circ} \mathrm{C}$ with the random seed of 1420883531.

Root mean square deviation (RMSD), which characterizes the amount of simulated molecule deviates from their defined positions in space, is calculated by program using the equation:

$$
\operatorname{RMSD}_{a}\left(t_{j}\right)=\sqrt{\frac{\sum_{a=1}^{N_{a}}\left(r_{a}\left(t_{j}\right)-r_{a}\right)^{2}}{N_{a}}}
$$

where $N_{a}$ is the number of atoms whose positions are compared, $t_{j}$ is time, $r_{a}\left(t_{j}\right)$ is the position of atom $a$ at tine $t_{p}<$ ra $>$ is the average value of the position of atom $a$, to which the position $r_{a}\left(t_{j}\right)$ is compared [18].

\section{Results}

It is important to study the mechanism of how $\alpha$-helical structure can spontaneously unfold and refold, considering its significance in clinical and biotechnical settings. In this study, the unfolding and refolding of plantaricin A were conducted in molecular dynamics simulation, because of its ready switch between folded and unfolded $\alpha$-helical structures.

Figure 1(a) demonstrates the unfolding process of $\alpha$-helix at $1 \mathrm{~ns}$ interval. As can be seen, the unfolding of $\alpha$-helix is not monotonic since it progresses faster between 0 and $1 \mathrm{~ns}$, and between 4 and 5 ns than other intervals. It is not surprising that the unfolding is faster between 0 and 1 ns because many studies showed the great role of the initial phase in molecular dynamics simulations. This is due to the change from the measured structure under a particular experimental condition to the condition of molecular dynamics simulation [19]. In addition, the unfolding did not begin at the termini of $\alpha$-helix, but began in its middle as seen in panels $0 \mathrm{~ns}$ and $1 \mathrm{~ns}$. 

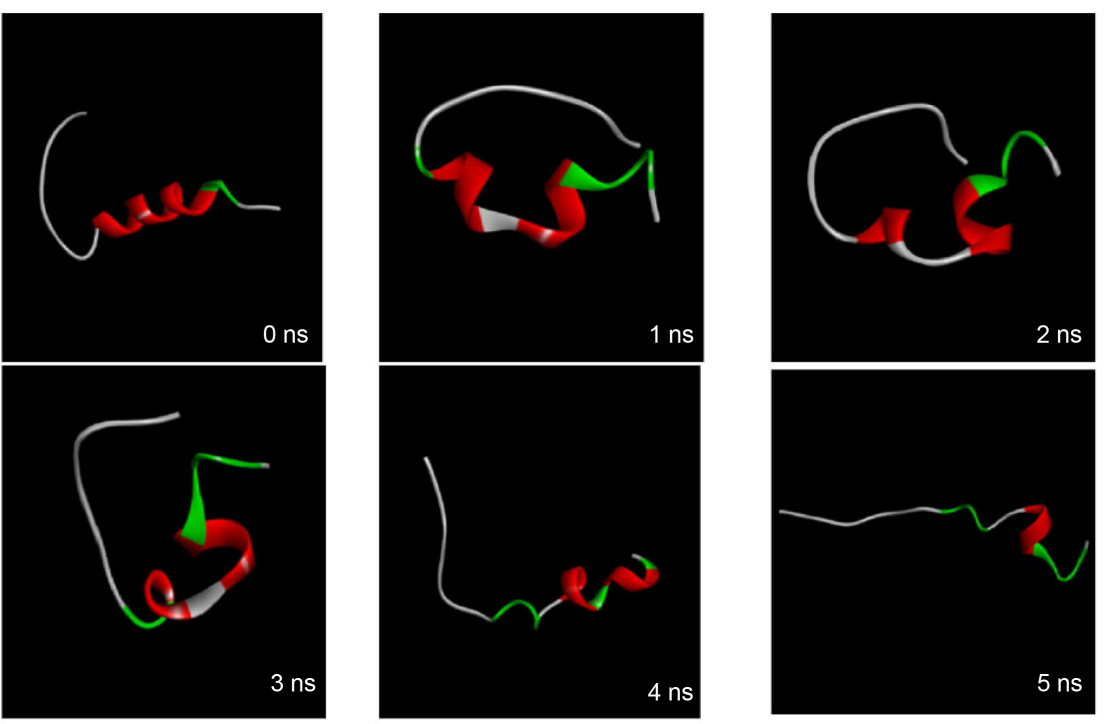

(a)
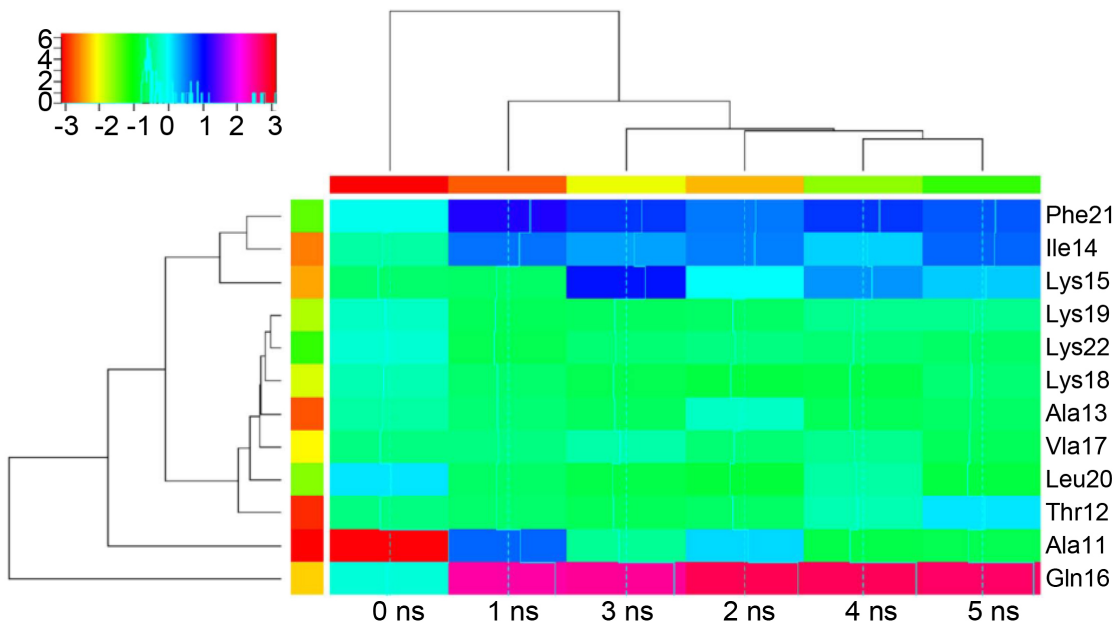

(b)

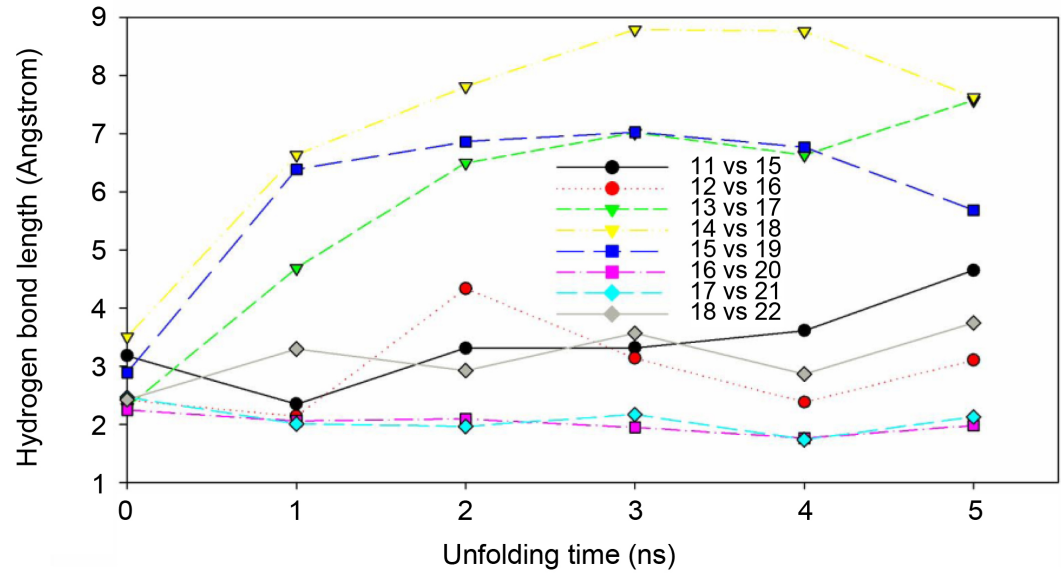

(c)

Figure 1. The unfolding of $\alpha$-helix of plantaricin A at 1 ns interval (a), the distance of the residues from position 11 to position 22 to the ideal $\alpha$-helical center $-60^{\circ}$ in $\phi$-axis and $-50^{\circ}$ in $\psi$-axis and their dendrogram produced by the cluster analysis during unfolding process (b), and the length of hydrogen bond between the residues $i$ and $i+4$ (c). 
In Figure 1(b), different colors illustrate how far away each residue moved away from the reference point, which is the ideal $\alpha$-helical center $-60^{\circ}$ in $\phi$-axis and $-50^{\circ}$ in $\psi$-axis in terms of the Ramachandran notation. As can be seen, the residues with green color did not have great movements from the reference point because their color did not change over $5 \mathrm{~ns}$, so these residues could not lead the $\alpha$-helix to unfold. On the contrary, the residues on the top and the bottom in Figure 1(b) moved away from the reference point because of changes in their colors, especially the glutamine at position 16. Meanwhile, the dendrogram produced by the cluster analysis suggests that this residue was the main force for the unfolding of the $\alpha$-helix. Moreover the alanine at position 11 appeared the main force to maintain the $\alpha$-helix because it moved towards the reference point over the time. This is reasonable because alanine is too short to effectively mediate its degradation [4]. Still, Figure 1(b) indicates that the turning point of unfolding of $\alpha$-helix is the time between 3 ns and 4 ns, because most residues actually move back to the reference point at 3 ns. In effect, the $\alpha$-helix will be refolded if such a tendency holds on.

Figure 1(c) demonstrated the length of hydrogen bonds between the residues $i$ and $i+4$ of $\alpha$-helix. As can be seen, the hydrogen bonds between residues 14 and 18 , between residues 15 and 19 , and between residues 13 and 17 increased more than others. These residues, isoleucine, lysine, glutamine, valine, lysine and lysine, have similar helix propensities except for valine [20]. Just two hydrogen bonds, between residues 16 and 20, between residues 17 and 21, hold their length around $2 \AA$, which is the typical length of a hydrogen bond [21].

The simulation in at $0 \mathrm{~ns}$ and 5 in Figure 1(a) demonstrates the difference between folded and unfolded $\alpha$-helix of plantaricin A, and this difference marks the opening of $\alpha$-helical structure.

Because the start of refolding of plantaricin A was different in each simulation, we set the refolding time scale from 0 to 5 ns (Figure 2) in order to be identical with time scale in unfolding (Figure 1). Figure 2(a) depicts the refolding process of $\alpha$-helix of plantaricin A at 1 ns interval, where the refolding began from a very short $\alpha$-helix, which is generally considered as the folding nuclei [22]. The same explanation in Figure 1(b) and Figure 1(c) can be applied to Figure 2(b) and Figure 2(c). Collectively, alanine is the initial residue for refolding as it has showed to be the main force to maintain the $\alpha$-helix in Figure 1 (b).

Misfolded plantaricin A, which can be eliminated by DnaK and GroEL in bacteria [23], suggests the difficulty in folding of native structure, because each residue has several pathways to be folded. When a plantaricin A can find a correct pathway to fold, it reached its native structure in $6 \mathrm{~ns}$ (Figure 2(a)). This is an extremely short period of time if we consider the number of pathways that a protein can fold itself. According to HP model [24] [25] [26] [27], where a protein folds in a lattice, the number of possible folding pathways for 26-residue sequence in $3 \mathrm{D}$ HP model is $6 \times 5^{25}$ to find its native state in the worst case. 

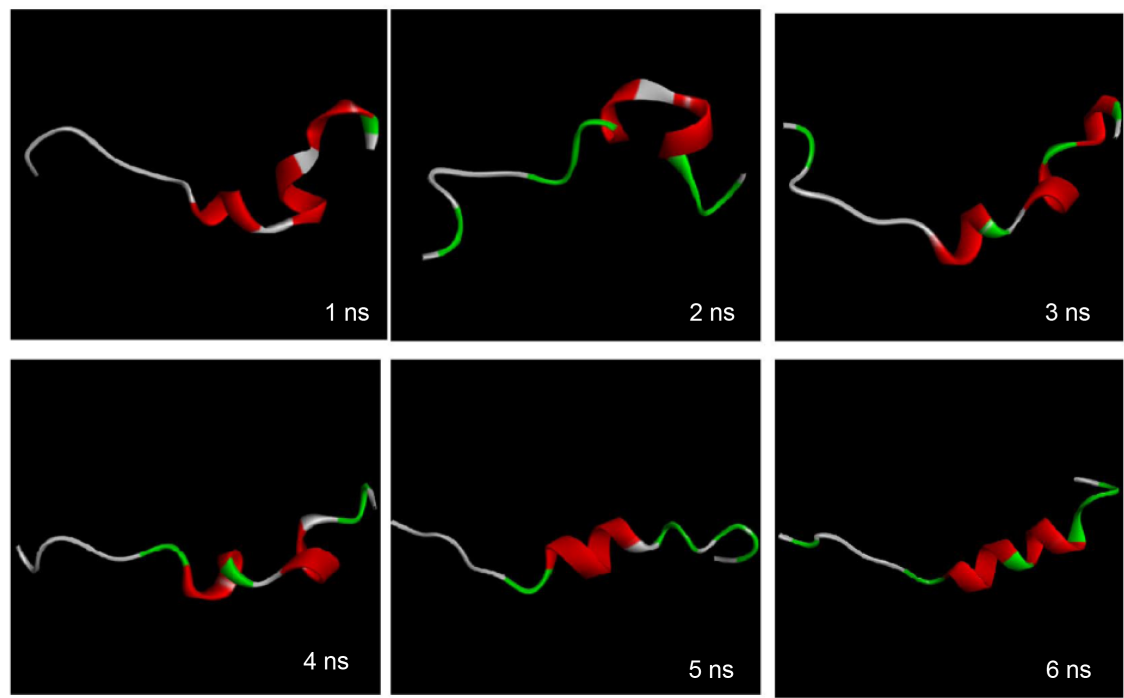

(a)
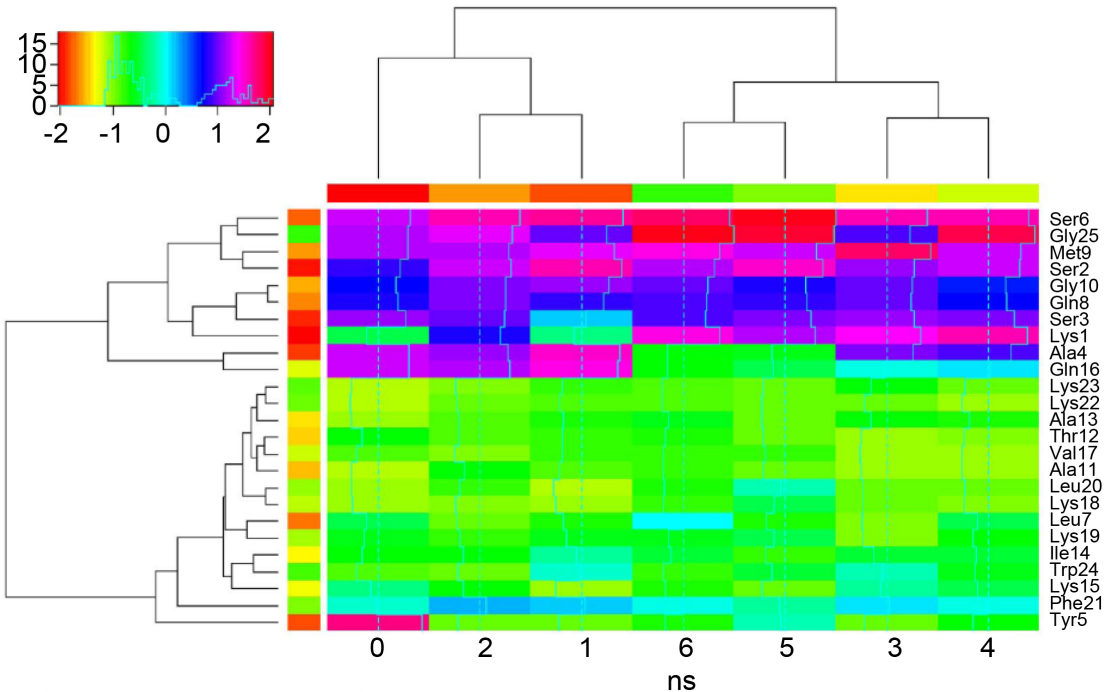

(b)

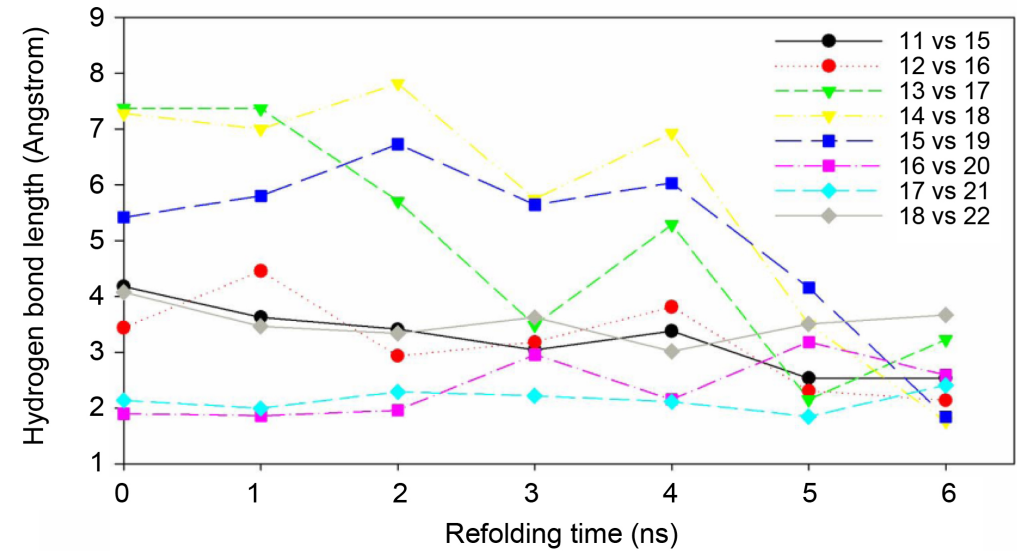

(c)

Figure 2. The refolding of $\alpha$-helix of plantaricin A at 1 ns interval (a), the distance of the residues from position 11 to position 22 to the ideal $\alpha$-helical center $-60^{\circ}$ in $\phi$-axis and $-50^{\circ}$ in $\psi$-axis and their dendrogram produced by the cluster analysis during refolding process (b), and the length of hydrogen bond between the residues $i$ and $i+4$ (c). 
The simulation in at $1 \mathrm{~ns}$ and 6 in Figure 2(a) shows the difference between unfolded and folded $\alpha$-helix of plantaricin A, and this difference marks the forming of $\alpha$-helical structure.

The native state of a protein is associated with the minimal energy. Molecular dynamics simulation provides the trajectories of atomic positions and energy over time for equilibration and minimization. Figure 3 reveals that plantaricin A needs roughly $5 \mathrm{~ns}$ to reach its equalization and minimization during refolding process. This period of time was used by plantaricin A to find the correct refolding pathway. It was suggested that the hydrophobic force is the main force determining the unique native state [24], while the percent of hydrophobic residues of majority of proteins is $40 \%-50 \%$ [28]. The percent of hydrophobic residues in plantaricin A is $42 \%$, so plantaricin A would follow this to fold itself.

Figure 4 represents the change in total energy of refolding of plantaricin A

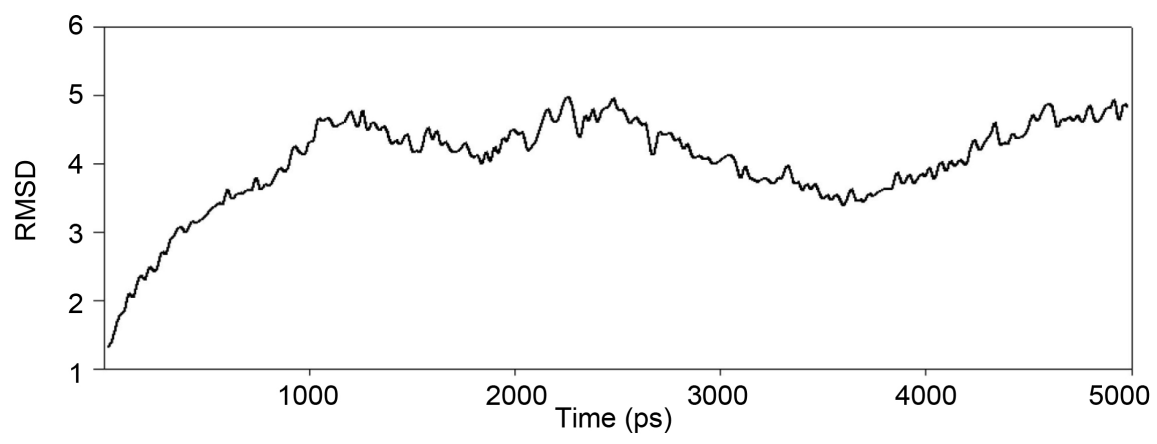

Figure 3. Root mean square deviation of refolding process of plantaricin A over time.

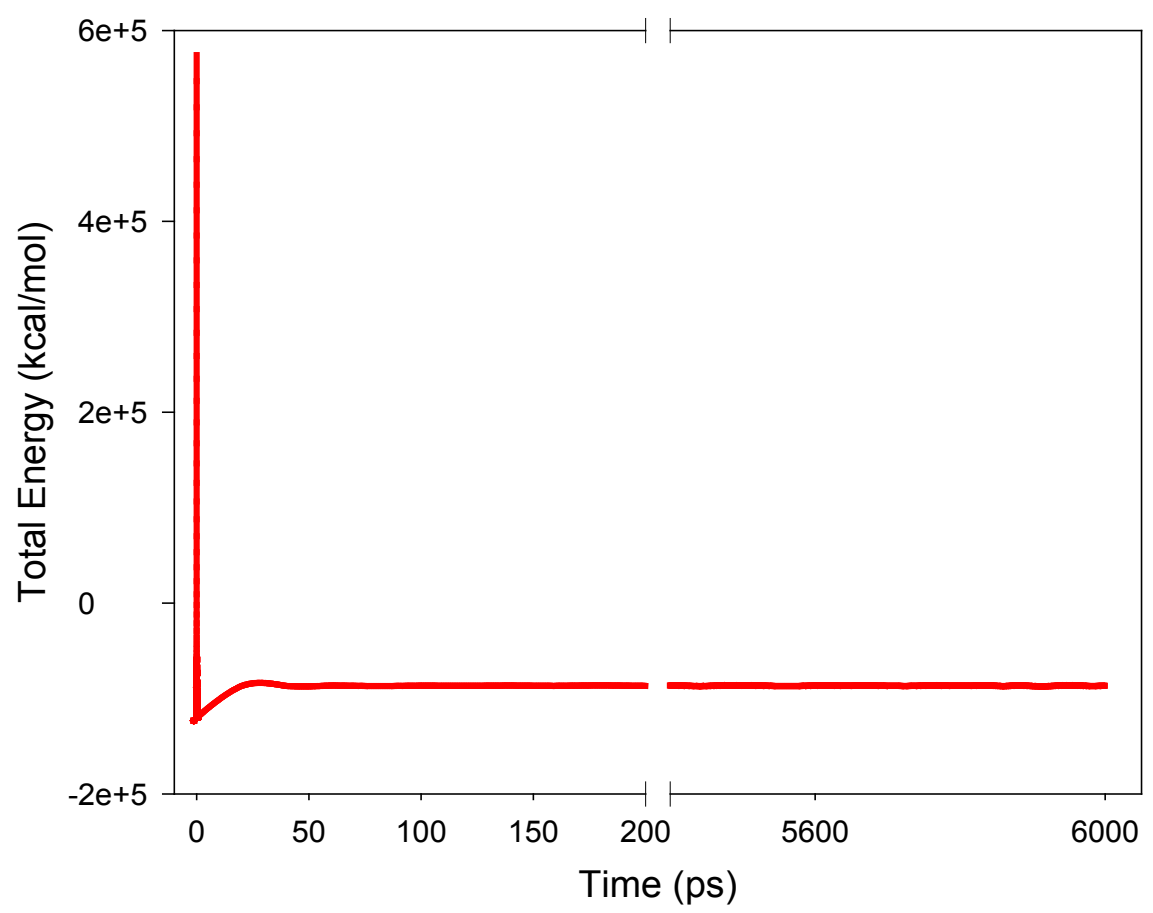

Figure 4. Change in total energy of refolding of plantaricin A during molecular dynamics simulation. 
during molecular dynamics simulation. At time 0 , there should be $100 \%$ unfolded structure and the total energy is at maximum. From 0 ps to $20 \mathrm{ps}$, the total energy decreased from $574,306 \mathrm{kcal} / \mathrm{mol}$ to $-120,017 \mathrm{kcal} / \mathrm{mol}$, while from 20 ps to 6000 ps the total energy fluctuated between $-120,000$ and $-80,000 \mathrm{kcal} / \mathrm{mol}$. These rapid decline in total energy suggests an energy funnel for folding [29] [30].

\section{Discussion}

Plantaricin A was chosen because it comes from Lactobacillus plantarum. It is known that bacteria evolved a mechanism to use proteins such as DnaK and GroEL to prevent the accumulation of unfolded and misfolded proteins [23]. Additionally, the folding of plantaricin A occurs at co-translational stage [31]. This is very delicate because the folding of proteins occurs either at the posttranslational stage or at the co-translational stage with different requirement of time. Thus, a peptide sequence must fold itself into a well-defined functional structure within a reasonable period of time. Essentially, the signal peptide of protein has the information on timing of co-translational stage [32], which is the case for plantaricin A [31].

The most important observation in the unfolding of $\alpha$-helix of plantaricin A is that the $\alpha$-helix is unfolded from the middle of $\alpha$-helix rather than from its termini. Theoretically, the breaking of hydrogen bond requires $30 \mathrm{~kJ} / \mathrm{mol}$ energy, which could be possible under the fluctuation in the system [33]. Hence the spontaneous unfolding of $\alpha$-helix is very probably to occur in vivo because molecular dynamics simulation with the artificial periodicity stabilizes a protein otherwise it would unfold quickly [34]. A study on the folding of DNA loop revealed that the required energy was roughly the same for misfolded and native loops [35].

For many proteins, their unfolding is irreversible, for example, the coiled-coil structure is irreversible in Hv1/VSOP [36]. Plantaricin A can refold itself again when exposing to micelles and under the physiological condition [11]. In molecular dynamic simulation, the refolding of plantaricin A is not easy with unspecific time of simulation, and the most important point is that most refolded structures are misfolding. A large number of misfoldings is understandable because the unfolding does not have many choices for each residue, but the folding of protein is far more complex, which is explained at least by three theories. 1) The hydrophobic-polar (HP) model [24] and the hydrophobic-hydrophilicneutral (BPN) model [37] enumerate all the possible folding pathways in order to find out the optimal pathway to rapidly fold the native structure. 2) The theory focuses on folding intermediates, and analyzes the stability and activation barriers between folding intermediates with folding reaction [38]. 3) The theory concentrates on folding energy landscape and proposes a rugged funnel-like landscape biased toward the native structure [29] [30]. Over years, the first and third theories are becoming more and more complementary [39]. 
Generally, the folding is faster for $\alpha$-helix than for $\alpha$-sheet as well as the mixture of $\alpha$-helix and $\alpha$-sheet [40], and plantaricin A belongs to the small ultrafast-folding proteins.

How a protein can find out its native state without globally exhaustive search is the Levinthal paradox [41], which extends beyond the protein folding into other combinatorial problems in biological fields such as protein-protein interaction [42] and biomolecular complex assembly [43].

In the past, molecular dynamics simulation was used to unfold proteins with external forces, including mechanic force [44], chemical force [45], high temperature [46], pressure [47], light/electromagnetic radiation [48], whereas the simulation of refolding of proteins was also studied with low temperature [49]. In this study, we simulated the spontaneously unfolding and refolding of plantaricin $\mathrm{A}$, which is the advantage of our study because we did not apply any foreign force and environmental conditions. Molecular dynamics simulation demonstrates that plantaricin A unfolds its $\alpha$-helix from the middle residue, refolds its $\alpha$-helix from a terminal. Moreover, our study reveals that fluctuation of energy under the physiological condition is sufficient to initiate unfolding of plantaricin $\mathrm{A}$.

\section{Fund}

This study was supported by National Natural Science Foundation of China (31460296 and 31560315), Key Project of Guangxi Scientific Research and Technology Development Plan (AB17190534) and Special Funds for Building of Guangxi Talent Highland.

\section{Conflicts of Interest}

The authors declare no conflicts of interest regarding the publication of this paper.

\section{References}

[1] Reddy, K.V., Yedery, R.D. and Aranha, C. (2004) Antimicrobial Peptides: Premises and Promises. International Journal of Antimicrobial Agents, 24, 536-547. https://doi.org/10.1016/j.ijantimicag.2004.09.005

[2] Mahlapuu, M., Håkansson, J., Ringstad, L. and Björn, C. (2016) Antimicrobial Peptides: An Emerging Category of Therapeutic Agents. Frontiers in Cellular and Infection Microbiology, 6, 194. https://doi.org/10.3389/fcimb.2016.00194

[3] Takahashi, D., Shukla, S.K., Prakash, O. and Zhang, G. (2010) Structural Determinants of Host Defense Peptides for Antimicrobial Activity and Target Cell Selectivity. Biochimie, 92, 1236-1241. https://doi.org/10.1016/j.biochi.2010.02.023

[4] Aguda, A.H., Panwar, P., Du, X., Nguyen, N.T., Brayer, G.D. and Brömme, D. (2014) Structural Basis of Collagen Fiber Degradation by Cathepsin K. Proceedings of the National Academy of Sciences of the United States of America, 111, 1747417479. https://doi.org/10.1073/pnas.1414126111

[5] Pasupuleti, M., Schmidtchen, A. and Malmsten, M. (2012) Antimicrobial Peptides: key Components of the Innate Immune System. Critical Reviews in Biotechnology, 
32, 143-171. https://doi.org/10.3109/07388551.2011.594423

[6] Wang, G., Li, X. and Wang, Z. (2016) APD3: The Antimicrobial Peptide Database as a Tool for Research and Education. Nucleic Acids Research, 44, D1087-D1093. https://doi.org/10.1093/nar/gkv1278

[7] Nguyen, L.T., Haney, E.F. and Vogel, H.J. (2011) The Expanding Scope of Antimicrobial Peptide Structures and Their Modes of Action. Trends in Biotechnology, 29, 464-472. https://doi.org/10.1016/j.tibtech.2011.05.001

[8] Sarroukh, R., Cerf, E., Derclaye, S., Dufrêne, Y.F., Goormaghtigh, E., Ruysschaert, J.M. and Raussens, V. (2011) Transformation of Amyloid $\beta$ (1-40) Oligomers into Fibrils Is Characterized by a Major Change in Secondary Structure. Cellular and Molecular Life Sciences, 68, 1429-1438. https://doi.org/10.1007/s00018-010-0529-x

[9] Stephenson, K., Carter, N.M., Harwood, C.R., Petit-Glatron, M.F. and Chambert, R. (1998) The Influence of Protein Folding on Late Stages of the Secretion of Alpha-Amylases from Bacillus subtilis. FEBS Letters, 430, 385-389. https://doi.org/10.1016/S0014-5793(98)00698-X

[10] Yan, S. and Wu, G. (2017) Bottleneck in Secretion of $\alpha$-Amylase in Bacillus subtilis. Microbial Cell Factories, 16, 124. https://doi.org/10.1186/s12934-017-0738-1

[11] Nissen-Meyer, J., Larsen, A.G., Sletten, K., Daeschel, M. and Nes, I.F. (1993) Purification and Characterization of Plantaricin A, a Lactobacillus plantarum Bacteriocin Whose Activity Depends on the Action of Two Peptides. Journal of General Microbiology, 139, 1973-1978. https://doi.org/10.1099/00221287-139-9-1973

[12] Kinjo, A.R., Bekker, G.J., Suzuki, H., Tsuchiya, Y., Kawabata, T., Ikegawa, Y. and Nakamura, H. (2017) Protein Data Bank Japan (PDBj): Updated User Interfaces, Resource Description Framework, Analysis Tools for Large Structures. Nucleic Acids Research, 45, D282-D288. https://doi.org/10.1093/nar/gkw962

[13] Kristiansen, P.E., Fimland, G., Mantzilas, D. and Nissen-Meyer, J. (2005) Structure and Mode of Action of the Membrane-Permeabilizing Antimicrobial Peptide Pheromone Plantaricin A. Journal of Biological Chemistry, 280, 22945-22950. https://doi.org/10.1074/jbc.M501620200

[14] Humphrey, W., Dalke, A. and Schulten, K. (1996) VMD-Visual Molecular Dynamics. Journal of Molecular Graphics and Modelling, 14, 33-38. https://doi.org/10.1016/0263-7855(96)00018-5

[15] Phillips, J.C., Braun, R., Wang, W., Gumbart, J., Tajkhorshid, E., Villa, E., Chipot, C., Skeel, R.D., Kalé, L. and Schulten, K. (2005) Scalable Molecular Dynamics with NAMD. Journal of Computational Chemistry, 26, 1781-1802. https://doi.org/10.1002/jcc.20289

[16] MacKerell, Jr. A.D., Bashford, D., Bellott, M., Dunbrack, R.L., Evanseck, J.D., Field, M,J., Fischer, S., Gao, J., Guo, H., Ha, S., Joseph-McCarthy, D., Kuchnir, L., Kuczera, K., Lau, F.T., Mattos, C., Michnick, S., Ngo, T., Nguyen, D.T., Prodhom, B., Reiher, W.E., Roux, B., Schlenkrich, M., Smith, J.C., Stote, R., Straub, J., Watanabe, M., Wiórkiewicz-Kuczera, J., Yin, D. and Karplus, M. (1998) All-Atom Empirical Potential for Molecular Modeling and Dynamics Studies of Proteins. Journal of Physical Chemistry B, 102, 3586-3616. https://doi.org/10.1021/jp973084f

[17] Park, S. and Saven, J.G. (2006) Simulation of pH-Dependent Edge Strand Rearrangement in Human $\beta$-2 Microglobulin. Protein Science, 15, 200-207. https://doi.org/10.1110/ps.051814306

[18] University of Illinois at Urbana-Champaign, NIH Resource for Macromolecular Modeling and Bioinformatics Bechman Institute, NAMD Tutorial Windows Version. http://www.ks.uiuc.edu/Training/Tutorials/ 
[19] Shrivastava, I.H. and Sansom, M.S. (2000) Simulations of Ion Permeation through a Potassium Channel: Molecular Dynamics of KcsA in a Phospholipid Bilayer. Biophysical Journal, 78, 557-570. https://doi.org/10.1016/S0006-3495(00)76616-1

[20] Pace, C.N. and Scholtz, J.M. (1998) A Helix Propensity Scale Based on Experimental Studies of Peptides and Proteins. Biophysical Journal, 75, 422-427. https://doi.org/10.1016/S0006-3495(98)77529-0

[21] Legon, A.C. and Millen, D.J. (1987) Angular Geometries and Other Properties of Hydrogen-Bonded Dimers: A Simple Electrostatic Interpretation of the Success of the Electron-Pair Model. Chemical Society Reviews, 16, 467. https://doi.org/10.1039/cs9871600467

[22] Creighton, T.E. (1992) Protein Folding up the Kinetic Pathway. Nature, 356, 194-195. https://doi.org/10.1038/356194a0

[23] Wegele, H., Müller, L. and Buchner, J. (2004) Hsp70 and Hsp90-A Relay Team for Protein Folding. Reviews of Physiology Biochemistry and Pharmacology, 151, 1-44. https://doi.org/10.1007/s10254-003-0021-1

[24] Lau, K.F. and Dill, K.A. (1989) A Lattice Statistical Mechanics Model of the Conformation and Sequence Spaces of Proteins. Macromolecules, 22, 3986-3997. https://doi.org/10.1021/ma00200a030

[25] Yan, S. and Wu, G. (2012) Analysis on Folding of Misgurin Using 2-Dimensional HP Model. Proteins. Structure, Function, and Bioinformatics, 80, 764-773. https://doi.org/10.1002/prot.23233

[26] Yan, S. and Wu, G. (2012) Detailed Folding Structures of Kappa-Conotoxin RIIIJ and Its Mutageneses Obtained from 2-Dimensional HP Model. Protein \& Peptide Letters, 19, 567-572. https://doi.org/10.2174/092986612800190982

[27] Yan, S. and Wu, G. (2012) Detailed Folding Structures of M-Lycotoxin-Hcla and Its Mutageneses Using 2-Dimensional HP Model. Molecular Simulation, 38, 809-822. https://doi.org/10.1080/08927022.2012.654473

[28] Nandi, T., B-Rao, C. and Ramachandran, S. (2002) Comparative Genomics Using Data Mining Tools. Journal of Biosciences, 27, 15-25. https://doi.org/10.1007/BF02703680

[29] Leopold, P.E., Montal, M. and Onuchic, J.N. (1992) Protein Folding Funnels: A Kinetic Approach to the Sequence-Structure Relationship. Proceedings of the National Academy of Sciences of the United States of America, 89, 8721-8725. https://doi.org/10.1073/pnas.89.18.8721

[30] Onuchic, J.N., Luthey-Schulten, Z. and Wolynes, P.G. (1997) Theory of Protein Folding: The Energy Landscape Perspective. Annual Review of Physical Chemistry, 48, 545-600. https://doi.org/10.1146/annurev.physchem.48.1.545

[31] Zhao, H., Sood, R., Jutila, A., Bose, S., Fimland, G., Nissen-Meyer, J. and Kinnunen, P.K. (2006) Interaction of the Antimicrobial Peptide Pheromone Plantaricin A with Model Membranes: Implications for a Novel Mechanism of Action. Biochimica et Biophysica Acta, 1758, 1461-1474. https://doi.org/10.1016/j.bbamem.2006.03.037

[32] Rutkowski, D.T., Ott, C.M., Polansky, J.R. and Lingappa, V.R. (2003) Signal Sequences Initiate the Pathway of Maturation in the Endoplasmic Reticulum Lumen. Journal of Biological Chemistry, 278, 30365-30372.

https://doi.org/10.1074/jbc.M302117200

[33] Chan, H.S., Zhang, Z., Wallin, S. and Liu, Z.R. (2011) Cooperatively, Local-Nonlocal Coupling, and Nonnative Interactions: Principles of Protein Folding from Coarse-Grained Models. Annual Review of Physical Chemistry, 62, 301-326.

https://doi.org/10.1146/annurev-physchem-032210-103405 
[34] Weber, W., Hünenberger, P.H. and McCammon, J.A. (2000) Molecular Dynamics Simulations of a Polyalanine Octapeptide under Ewald Boundary Conditions: Influence on Artificial Periodicity of Peptide Conformation. Journal of Physical Chemistry B, 104, 3668-3675. https://doi.org/10.1021/jp9937757

[35] Ansari, A., Kuznetsov, S.V. and Shen, Y. (2001) Configurational Diffusion down a Folding Funnel Describes the Dynamics of DNA Hairpins. Proceedings of the National Academy of Sciences of the United States of America, 98, 7771-7776. https://doi.org/10.1073/pnas.131477798

[36] Fujiwara, Y., Kurokawa, T., Takeshita, K., Kobayashi, M., Okochi, Y., Nakagawa, A. and Okamura, Y. (2012) The Cytoplasmic Coiled-Coil Mediates Cooperative Gating Temperature Sensitivity in the Voltage-Gated H (+) Channel Hvl. Nature Communications, 3, 816. https://doi.org/10.1038/ncomms1823

[37] Nymeyer, H., García, A.E. and Onuchic, J.N. (1998) Folding Funnels and Frustration in Off-Lattice Minimalist Protein Landscapes. Proceedings of the National Academy of Sciences of the United States of America, 95, 5921-5828. https://doi.org/10.1073/pnas.95.11.5921

[38] Laurents, D.V. and Baldwin, R.L. (1998) Protein Folding: Matching Theory and Experiment. Biophys Journal, 75, 428-434. https://doi.org/10.1016/S0006-3495(98)77530-7

[39] Dill, K.A. and MacCallum, J.L. (2012) The Protein-Folding Problem, 50 Years on. Science, 338, 1042-1046. https://doi.org/10.1126/science.1219021

[40] Lindorff-Larsen, K., Piana, S., Dror, R.O. and Shaw, D.E. (2011) How Fast-Folding Proteins Fold. Science, 334, 517-520. https://doi.org/10.1126/science.1208351

[41] Levinthal, C. (1968) Are There Pathways for Protein Folding? Journal de Chimie Physique et de Physico-Chimie Biologique, 65, 44-45. https://doi.org/10.1051/jcp/1968650044

[42] Tompa, P. and Rose, G.D. (2011) The Levinthal Paradox of the Interactome. Protein Science, 20, 2074-2079. https://doi.org/10.1002/pro.747

[43] Zenk, J. and Schulman, R. (2004) An Assembly Funnel Makes Biomolecular Complex Assembly Efficient. PLOS ONE, 9, e111233. https://doi.org/10.1371/journal.pone.0111233

[44] Gräter, F., Shen, J., Jiang, H., Gautel, M. and Grubmüller, H. (2005) Mechanically Induced Titin Kinase Activation Studied by Force-Probe Molecular Dynamics Simulations. Biophysical Journal, 88, 790-804. https://doi.org/10.1529/biophysj.104.052423

[45] Stumpe, M.C. and Grubmüller, H. (2007) Aqueous Urea Solutions: Structure, Energetics, and Urea Aggregation. Journal of Physical Chemistry B, 111, 6220-6228. https://doi.org/10.1021/jp066474n

[46] Steel, B.C., McKenzie, D.R., Bilek, M.M., Nosworthy, N.J. and dos Remedios, C.G. (2006) Nanosecond Responses of Proteins to Ultrahigh Temperature Pulses. Biophysical Journal, 91, L66-L68. https://doi.org/10.1529/biophysj.106.090944

[47] Schäfer, L.V., Müller, E.M., Gaub, H.E. and Grubmüller, H. (2007) Elastic Properties of Photoswitchable Azobenzene Polymers from Molecular Dynamics Simulations. Angewandte Chemie International Edition, 46, 2232-2237. https://doi.org/10.1002/anie.200604595

[48] Larios, E., Li, J.S., Schulten, K., Kihara, H. and Gruebele, M. (2004) Multiple Probes Reveal a Native-Like Intermediate during Low-Temperature Refolding of Ubiquitin. Journal of Molecular Biology, 340, 115-125. https://doi.org/10.1016/j.jmb.2004.04.048 
[49] Liu, Y., Prigozhin, M.B., Schulten, K. and Gruebele, M. (2014) Observation of Complete Pressure-Jump Protein Refolding in Molecular Dynamics Simulation and Experiment. Journal of the American Chemical Society, 136, 4265-4272.

https://doi.org/10.1021/ja412639u 